\title{
Understanding interactions between capped nanocrystals: Three-body and chain packing effects
}

\author{
Philipp Schapotschnikow and Thijs J. H. Vlugt ${ }^{\text {a) }}$ \\ Process and Energy Laboratory, Delft University of Technology, Leeghwaterstraat 44, \\ 2628 CA Delft, The Netherlands
}

(Received 16 June 2009; accepted 24 August 2009; published online 23 September 2009)

\begin{abstract}
Self-assembly of capped nanocrystals (NC) attracted a lot of attention over the past decade. Despite progress in manufacturing of NC superstructures, the current understanding of their mechanical and thermodynamic stability is still limited. For further applications, it is crucial to find the origin and the magnitude of the interactions that keep self-assembled NCs together, and it is desirable to find a way to rationally manipulate these interactions. We report on molecular simulations of interacting gold NCs protected by capping molecules. We computed the potential of mean force for pairs and triplets of NCs of different size $(1.8-3.7 \mathrm{~nm})$ with varying ligand length (ethanethiol-dodecanethiol) in vacuum. Pair interactions are strongly attractive due to attractive van der Waals interactions between ligand molecules. Three-body interaction results in an energy penalty when the capping layers overlap pairwise. This effect contributes up to $20 \%$ to the total energy for short ligands. For longer ligands, the three-body effects are so large that formation of NC chains becomes energetically more favorable than close packing of capped NCs at low concentrations, in line with experimental observations. To explain the equilibrium distance for two or more NCs, the overlap cone model is introduced. This model is based on relatively simple ligand packing arguments. In particular, it can correctly explain why the equilibrium distance for a pair of capped NCs is always $\approx 1.25$ times the core diameter independently on the ligand length, as found in our previous work [Schapotschnikow, R. Pool, and T. J. H. Vlugt, Nano Lett. 8, 2930 (2008)]. We make predictions for which ligands capped NCs self-assemble into highly stable three-dimensional structures, and for which they form high-quality monolayers. (C) 2009 American Institute of Physics.
\end{abstract}

[doi:10.1063/1.3227043]

\section{INTRODUCTION}

Gold and semiconductor nanocrystals (NCs) with specific size and shape dependent optical and electrical behavior are of growing importance in the fields of optics, electronics, catalysis, magnetic storage, and biophysics. ${ }^{1}$ These NCs can self-assemble in a range of different two-dimensional (2D) and three-dimensional (3D) superstructures. ${ }^{2-5}$ NCs are usually protected by an organic capping layer that prevents aggregation, e.g., gold NCs are often capped with alkyl thiol molecules. ${ }^{6}$ These capping molecules (also referred to as surfactants or ligands) play an important role in the selfassembly of NCs. The ratio between the core diameter $\left(d_{c}\right)$ and surfactant chain length $(L)$ determines the crystal structure of a Au NC superlattice. ${ }^{5,7}$ Capping exchange can alter the crystal structure of a binary superlattice. ${ }^{8}$ Moreover, due to the attractive interactions between capping molecules, in binary NC systems, crystal structures with high coordination form rather than the ones with a higher packing fraction. The attraction between capped NCs can be very strong; for example, a monolayer of Au NCs can form a self-supported membrane when dropcasted on a substrate with a hole up to $2 \mu \mathrm{m}$ large. ${ }^{10}$ It is well known that this attraction is at least one order of magnitude larger than the van der Waals (vdW) attractions between $\mathrm{Au} \mathrm{NC}$ cores. ${ }^{10-12}$

${ }^{a)}$ Electronic mail: t.j.h.vlugt@tudelft.nl.
For the understanding of thermodynamic and kinetic properties of NCs, knowledge of the free energy or, equivalently, the potential of mean force (PMF) as a function of an appropriate order parameter is of vital importance. ${ }^{13,14}$ This would allow fabrication of novel materials and devices with tailor-made structural, mechanical, and thermodynamic properties. The objective of this work is to study the effective interactions between capped NCs in vacuum. Molecular simulation techniques provide an excellent tool for the computation of these, especially for nanoscale systems, as these techniques do not suffer from experimental limitations or from oversimplifications sometimes present in theories. ${ }^{15}$ The computed PMFs are useful for several purposes. First, they provide direct insight in the mechanical and thermodynamic stability of different self-assembled structures. Second, these effective interactions can be used to model mechanical properties of NC superstructures. Third, the PMF in vacuum can be used to describe capped NCs at the air-water interface, as NCs capped by hydrophobic ligands are hardly immersed in water at the surface. It is important to note, however, that NC self-assembly is typically performed in solution and is therefore governed by interactions different from the ones presented in this paper. Therefore, the results of the present work can only be used to make statements about the properties of already formed NC superstructures.

Capped gold NCs are a convenient system for molecular 
simulation studies. The binding of thiol headgroups to $\mathrm{Au}$ surfaces is better understood than for other NC-surfactant systems. ${ }^{16}$ Several models have been successfully applied to describe the structure and thermodynamics of alkyl thiol monolayers on flat $\mathrm{Au}(111)$-surfaces ${ }^{17-19}$ and Au-NCs. ${ }^{18-21} \mathrm{It}$ is important to note that the effective NC-NC interactions in a solvent are very different from the ones in vacuum due to solvent-capping layer interactions. The work of Patel and Egorov $^{22}$ focused on the PMF for very small capped NC cores $\left(\mathrm{Au}_{38}\right)$ for varying solvent quality, and it was found that the PMF can be tuned from strongly attractive to fully repulsive. In our previous work, we have shown for one system that the interaction between alkylthiol capped NCs becomes purely repulsive in a good solvent (n-hexane), ${ }^{23}$ we are currently investigating in detail the NC-NC interactions in different solvents at different conditions. Tay and Bresme $^{12}$ computed the PMF for only two selected systems in vacuum so that no definitive conclusions could be drawn concerning the general behavior of the PMF.

In our previous work, ${ }^{23}$ we systematically investigated the dependence of the PMF on several crucial parameters, such as capping length, NC size, and temperature. For all systems we studied, we found that, surprisingly, the equilibrium distance (i.e., the minimum of the $\mathrm{PMF}$ ) for a pair of capped NCs is $\approx 1.25$ times the NC core diameter, independently on the chain length. In the present work, we study for the first time triplet interactions between capped NCs. The computed PMF between pairs or triplets of NCs can be understood quantitatively by the Overlap Cone Model, which we will present here. This model is based on chain packing arguments. Based on the computed PMFs, we develop a coarse-grained NC-NC interaction potential.

The remainder of this paper is structured as following. Section II contains a description of the model and methods used in this work. In Sec. III, we briefly discuss our simulation results for two-body interactions. Effective three-body interactions are quantified in Sec. IV. The computed effective interactions are parametrized in Secs. III A and IV A, so that they can be used in future studies. In Sec. V, we will introduce the OCM to understand the relation between chain packing effects and the PMF for pairs and triplets of NCs. In Sec. VI, we combine our findings to make a prediction which ligand is appropriate for which NC structure. Section VII summarizes the main results.

\section{MODEL AND METHODS}

In all simulations, we apply the united atom model for $\mathrm{SH}, \mathrm{CH}_{2}$, and $\mathrm{CH}_{3}$ groups. Alkylthiols are labeled as " $\mathrm{SC}_{n}$," where $n$ is the number of alkyl chain segments in the linear tail. Beads of different surfactant molecules interact with each other and with gold atoms via truncated and shifted Lennard-Jones (LJ) pair interactions; parameters are summarized in Table I. Note that the $\mathrm{Au}-\mathrm{S}$ interaction is much stronger than other nonbonded interactions. We account for intramolecular bond stretching, bond bending, and torsional interactions. ${ }^{24,25}$ Additionally, we apply intramolecular LJ in-
TABLE I. Force field parameters for the LJ interactions in our system. The $\mathrm{CH}_{x}-\mathrm{CH}_{y}$ interaction parameters are taken from Ref. 25. The S-CH${ }_{x}$ interactions are taken from Ref. 17. $\mathrm{Au}-\mathrm{S}$ and $\mathrm{Au}-\mathrm{CH}_{x}$ interactions are taken from our previous work (Refs. 18 and 19) Interactions between rigid NC cores are modeled via the Hamaker potential [Eq. (1)]. LJ interactions are truncated and shifted at $12 \AA$.

\begin{tabular}{lcccc}
\hline \hline$\epsilon_{i j} / k_{B}(\mathrm{~K})$ & $\mathrm{CH}_{3}$ & $\mathrm{CH}_{2}$ & $\mathrm{SH}$ & $\mathrm{Au}$ \\
\hline $\mathrm{CH}_{3}$ & 108 & 78 & 117 & 108 \\
$\mathrm{CH}_{2}$ & 78 & 56 & 84 & 88 \\
$\mathrm{SH}$ & 117 & 84 & 126 & 2795 \\
$\mathrm{Au}$ & 108 & 88 & 2795 & $\cdots$ \\
& & & & \\
$\sigma_{i j}(\AA)$ & $\mathrm{CH}_{3}$ & $\mathrm{CH}_{2}$ & $\mathrm{SH}$ & $\mathrm{Au}$ \\
\hline $\mathrm{CH}_{3}$ & 3.76 & 3.86 & 4.11 & 3.54 \\
$\mathrm{CH}_{2}$ & 3.86 & 3.96 & 4.21 & 3.54 \\
$\mathrm{SH}$ & 4.11 & 4.21 & 4.45 & 2.65 \\
$\mathrm{Au}$ & 3.54 & 3.54 & 2.65 & $\cdots$ \\
\hline \hline
\end{tabular}

teraction between segments that are separated by more than three bonds. Electrostatic interactions are not taken into account explicitly in our model.

The NC cores are modeled as rigid, close-packed icosahedra exposing only (111)-facets. ${ }^{26}$ The maximal extent $r_{\max }$ is defined as the center-to-corner distance, and the core diameter $d_{c}$ is defined as twice the radius of gyration. Gold NC cores are assumed to interact with each other via the Hamaker potential, ${ }^{27}$

$$
U_{\mathrm{Ham}}(r)=-\frac{A_{\mathrm{Ham}}}{12}\left[\frac{d_{c}^{2}}{r^{2}-d_{c}^{2}}+\frac{d_{c}^{2}}{r^{2}}+2 \ln \left(1-\frac{d_{c}^{2}}{r^{2}}\right)\right] .
$$

The interaction constant $A_{\mathrm{Ham}}$ is $2 \mathrm{eV}$ for gold in an apolar hydrocarbon medium. ${ }^{28}$ It should be noted that various alternatives to Eq. (1) for the effective vdW interactions between NC cores have been studied recently. ${ }^{29}$ These expressions deviate at most by a factor 2 from the Hamaker potential. We show at the beginning of Sec. III that such variations have no impact on our results as these interactions are very weak compared to the interactions between molecules in the capping layer.

In all simulations periodic boundary conditions are imposed. The simulation box is chosen sufficiently large, so that capping layers of NCs do not interact with their periodic images. Simulations are performed at constant temperature $T=300 \mathrm{~K}$. An overview of all simulations including their length is given in Table II.

\section{A. Potential of mean force}

Our aim is to determine the free energy, or, equivalently, the PMF as a function of NC-NC separation. We use constraint Monte Carlo (MC) and molecular dynamics (MD) methods for the computation of the PMF. Consider two NCs at the fixed distance $r$. The mean force $F_{\text {mean }}$ is defined as the average force between the two particles in direction of their connecting line: $:^{30,31}$ 
TABLE II. Overview of the PMF calculations in this work. Simulation length is expressed in time (ns) for MD and the number of cycles for MC simulations, respectively. A MC cycle consists of $N+10$ steps, where $N$ is the total number of (capping) molecules in the system. Numbers in brackets denote simulations lengths for close NC-NC separations where capping layers strongly overlap. All simulations were carried out in the NVT ensemble at $T=300 \mathrm{~K}$

\begin{tabular}{lcc}
\hline \hline System & Method & Length \\
\hline $\mathrm{Au}_{147}\left(\mathrm{SC}_{4}\right)_{58}$ & $\mathrm{MD}$ & 5 \\
$\mathrm{Au}_{147}\left(\mathrm{SC}_{8}\right)_{58}\left(\mathrm{SC}_{12}\right)_{58}$ & $\mathrm{MC}$ & $1.5(3) \times 10^{5}$ \\
$\mathrm{Au}_{147}\left(\mathrm{SC}_{4}\right)_{136}$ & $\mathrm{MD}$ & 10 \\
$\mathrm{Au}_{561}\left(\mathrm{SC}_{8}\right)_{136}$ & $\mathrm{MD}$ & $1(3)$ \\
$\mathrm{Au}_{561}\left(\mathrm{SC}_{12}\right)_{136}$ & $\mathrm{MC}$ & $1.5(3) \times 10^{5}$ \\
$\mathrm{Au}_{561}\left(\mathrm{SC}_{4}\right)_{136}-\mathrm{Au}_{147}\left(\mathrm{SC}_{4}\right)_{58}$ & $\mathrm{MD}$ & $1(3)$ \\
$\mathrm{Au}_{561}\left(\mathrm{SC}_{8}\right)_{136}-\mathrm{Au}_{147}\left(\mathrm{SC}_{8}\right)_{58}$ & $\mathrm{MC}$ & $1(5) \times 10^{5}$ \\
$\mathrm{Au}_{561}\left(\mathrm{SC}_{145}\left(\mathrm{SC}_{2}\right)_{242}\right.$ & $\mathrm{MC}$ & $1(5) \times 10^{5}$ \\
$\mathrm{Au}_{141}\left(\mathrm{SC}_{3}\right)_{242}$ & $\mathrm{MD}$ & 1 \\
$\mathrm{Au}_{1415}\left(\mathrm{SC}_{6}\right)_{242}$ & $\mathrm{MD}$ & 1 \\
$\mathrm{Au}_{1415}\left(\mathrm{SC}_{12}\right)_{242}$ & $\mathrm{MD}$ & 1 \\
$\mathrm{Au}_{1415}\left(\mathrm{SC}_{4}\right)_{58}$ three-body & $\mathrm{MD}$ & $1.5(5) \times 10^{5}$ \\
$\mathrm{Au}_{147}\left(\mathrm{SC}_{8}\right)_{58}$ three-body & $\mathrm{MC}$ & $0.5(2) \times 10^{6}$ \\
$\mathrm{Au}_{147}$ & $\mathrm{MC}$ & \\
\hline \hline
\end{tabular}

$$
F_{\text {mean }}(r)=\frac{1}{2}\left\langle\left(\vec{F}_{2}-\vec{F}_{1}\right) \cdot \vec{r}_{u}\right\rangle_{\mathrm{NVT} ; r},
$$

where $\vec{F}_{1}$ and $\vec{F}_{2}$ are the total forces acting on the first and second NC core, respectively; $\vec{r}_{u}=\vec{r} / r$ is the unit vector connecting the two NCs, and angular brackets denote ensemble averages in the canonical ensemble with the constraint $\mathrm{NC}$ separation $r$. The PMF is defined as

$$
\phi_{\mathrm{MF}}(r)=\int_{r}^{\infty} F_{\text {mean }}(s) d s
$$

The mean force can be computed according to Eq. (2) using either constraint MC or MD simulations in the canonical ensemble. Equation (3) is then used to calculate the PMF. We experienced that the two methods have similar efficiency for simulations in vacuum, while MD is more efficient for simulations with explicit solvent. ${ }^{23}$

Alternatively, the PMF can be computed from unconstrained equilibrium $\mathrm{MD} / \mathrm{MC}$ simulations using, e.g., umbrella sampling with multiple-histogram reweighting, see Refs. 32-34 for recent examples. Steered molecular dynamics is a nonequilibrium method for the calculation of the PMF and it involves the averaging of nonreversible work performed during MD trajectories. ${ }^{35-38}$ In our previous work, we have shown that aggregates of capped NCs with interpenetrating capping layers feature shape memory: A transient restoring force acts upon small displacements of $\mathrm{NCs} .{ }^{23} \mathrm{As}$ transient forces do not influence thermodynamic (equilibrium) properties, they should not be accounted for in PMF computations. However, in our systems of interest, these forces act on time scales much longer than nanoseconds, so that results from unconstraint simulations may be misleading. In Ref. 23 we showed that constraint simulations suffer much less from this problem.

\section{Monte Carlo and molecular dynamics simulations}

In MC simulations, the degrees of freedom of surfactant molecules are sampled using displacement, ${ }^{39}$ rotation, ${ }^{40}$ and configurational-bias MC trial moves. ${ }^{41-44}$ The rotational degrees of freedom of the individual NCs are sampled using rotations of the NC core or of the cluster defined as the NC with ligands adsorbed on its surface. The centers of mass of the NCs remain fixed during the simulation.

The MD simulations are performed using the velocity Verlet (VV) algorithm. ${ }^{15,45}$ The only constraint on the system was applied to the NC-NC center of mass separation using the RATTLE algorithm, ${ }^{46}$ the VV version of SHAKE. ${ }^{47}$ Hereby, the NCs are "bonded" with a fixed length. The NC-NC bond can translate and rotate freely. The rotation of rigid NCs about their centers of mass is realized using quaternion rigid body dynamics. ${ }^{48}$ The temperature is kept constant using the Andersen thermostat. ${ }^{49}$

\section{B. Three-body interactions}

One of the difficulties in quantifying many-body effects is the number of internal coordinates involved. For a pair of (quasi-) isotropic objects (such as capped NCs in the present work), it is sufficient to consider the PMF as a function of only one parameter (here, $\mathrm{NC}$ center-to-center distance). In a system of three isotropic objects, one already needs three coordinates to describe all different configurations. If one wishes to explore all degrees of freedom of this system, the number of required simulations would increase cubically compared to a similar parametrization study of the corresponding two-body system. Without any additional information about the system, the computational costs required to parametrize three-body interactions are therefore much larger compared to a parametrization of isotropic pair interactions. If a three-body contribution to the total energy is necessary, one has to find a suitable potential form to make the computed interactions applicable in practice. This task is immensely simplified when one knows the nature of the interaction a priori. For instance, the energy of three consecutive beads in a chain molecule can be often decomposed into two bond stretching and one bond bending potentials. Unfortunately, such knowledge is not available for most soft-matter systems. Currently, simulation and theoretical studies mainly show presence or absence of many-body effects in one specific arrangement. ${ }^{50-53}$ In Refs. 50 and 53, e.g., the threebody force in a triplet of star polymers and dendrimers, respectively, was studied. The three objects were placed in an equilateral triangle, and mean force was computed as a function of the side length. It was concluded in Refs. 50 and 53 that three-body interactions are repulsive and have a significant contribution to the total energy. By contrast, an attractive three-body interaction was found for charged colloids in Ref. 52.

Our approach is to decompose the total interaction between three NCs into a sum of effective pair interactions computed for isolated $\mathrm{NC}$ pairs and a three-body correction term. Our simulation setup is sketched in Fig. 1(a). The centers $\mathrm{NC} 1, \mathrm{NC} 2$, and $\mathrm{NC} 3$ of three capped NCs are constraint to the corners of an isosceles triangle, where the NC1-NC3 

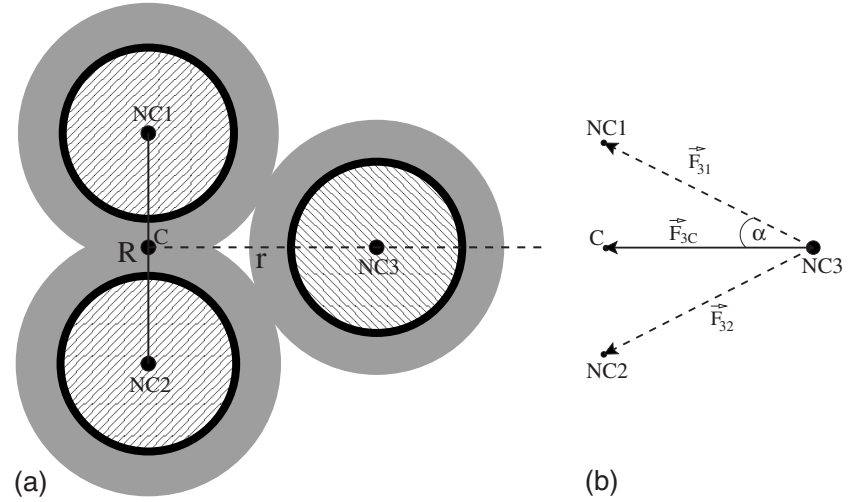

FIG. 1. (a) The setup of a constraint simulation of three NCs. NC cores are represented by shaded circles; the capping layers by gray rings. The centers of mass of the three NCs are constraint at positions denoted by $\mathrm{NC} 1, \mathrm{NC} 2$, and NC3, respectively. The midpoint between $\mathrm{NC} 1$ and $\mathrm{NC} 2$ is labeled as $C$. In a series of simulations, the distance $R=R_{12}$ between $\mathrm{NC} 1$ and NC2 is kept constant, while the distance $r=r_{3 C}$ between $C$ and NC3 varies. (b) Forces in the 3-NC system that are used to quantify the three-body effects. The mean force between NC3 and $C,\left|\vec{F}_{3 C}\right|$, is sampled from a constraint simulation. The effective forces between NC3 and NC1 (NC2) are denoted by $\vec{F}_{31}\left(\vec{F}_{32}\right)$. If no three-body interactions are present in the system, then the magnitudes of these effective forces are equal to the two-body mean force $F_{\text {mean }}$ at the corresponding distances.

and NC2-NC3 distances (denoted by $r_{13}$ and $r_{23}$, respectively) are equal. The degrees of freedom of ligand molecules and rotational degrees of freedom of the NCs are sampled using $\mathrm{MC}$ in the NVT ensemble. The mean force $F_{\text {mean }}\left(r_{3 C}\right)$ between $\mathrm{NC} 3$ and the NC1-NC2 midpoint $C$ is calculated from

$$
F_{\text {mean }}\left(r_{3 C}\right)=\frac{1}{2}\left\langle\left(\vec{F}_{C}-\vec{F}_{3}\right) \cdot \vec{r}_{u}\right\rangle_{\mathrm{NVT} ; R_{12}, r_{3 C}},
$$

where $\vec{F}_{C}$ and $\vec{F}_{3}$ are the total forces acting on $C$ and NC3, respectively; $r_{3 C}$ is the distance between these two points, and $\vec{r}_{u}=\vec{r}_{3 C} / r_{3 C}$ is the unit vector between them. The angular brackets denote an average in the canonical ensemble with constraint NC distances. This force can be decomposed into a vector sum of two forces, $\vec{F}_{13}$ and $\vec{F}_{23}$, acting between NC1 and NC3, and NC2 and NC3, respectively, see Fig. 1(b). The forces $\vec{F}_{13}$ and $\vec{F}_{23}$ are equal in magnitude by symmetry, so that we can define the effective two-body force $F_{\text {eff }}\left(r_{13}\right)$ in the three-body system as

$$
F_{\text {eff }}\left(r_{13}\right)=\left|\vec{F}_{13}\right|\left(r_{13}\right)=\left|\vec{F}_{13}\right|\left(r_{23}\right)=\frac{F_{\text {mean }}\left(r_{3 C}\right)}{2 \cos \alpha}
$$

with $r_{13}=r_{3 C} / \cos \alpha$. As shown in Fig. 1(b), $\alpha$ is the angle between the points $C, \mathrm{NC} 3$ and $\mathrm{NC} 1$ (or NC2). We perform a series of simulations in which the distance $R_{12}$ between $\mathrm{NC} 1$ and $\mathrm{NC} 2$ is kept constant, while $r_{3 C}$ is different in each simulation. Integrating the effective force $F_{\text {eff }}\left(r_{13}\right)$ with respect to the distance $r_{13}$, we obtain the effective PMF $\phi_{\mathrm{MF}}^{\text {eff }}\left(r_{13}\right)$,

$$
\phi_{\mathrm{MF}}^{\mathrm{eff}}\left(r_{13}\right)=\int_{r_{13}}^{\infty} F_{\text {eff }}\left(r^{\prime}\right) d r^{\prime}
$$

(a)

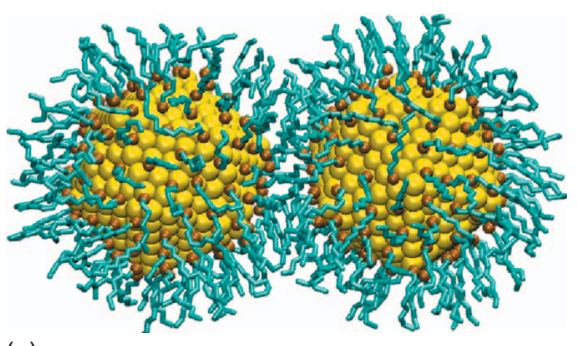

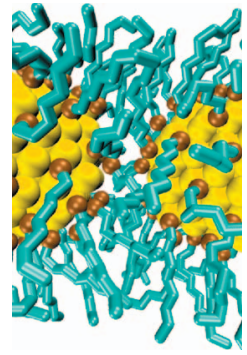

(b)
FIG. 2. (a) A pair of $\mathrm{Au}_{561} \mathrm{NCs}$ at $r=34 \AA$ capped by $136 \mathrm{SC}_{8}$ surfactant molecules. Yellow spheres represent gold atoms, brown spheres represent thiols headgroups, and blue lines represent alkyl tails. (b) Close-up of the "bottleneck" between NC surfaces. Note that the thiol capping molecules readily bend away from this bottleneck.

\section{Sample preparation}

The initial configurations for simulations in vacuum are prepared by a procedure similar to the one proposed in Ref. 20 . First, we generate a configuration with maximum surfactant coverage by a grand-canonical simulation of ethanethiol $\left(\mathrm{SC}_{2}\right)$ on a pair of NCs. The MC procedure described in Ref. 19 is used. Next, we perform MC simulations in the canonical ensemble at temperatures between 250 and $450 \mathrm{~K}$. Such extensive equilibration is important due to the slow diffusion of ligand headgroups on the NC surface. Finally, we exchange the $\mathrm{SC}_{2}$ ligand by the desired one using simulations in the semigrand ensemble, ${ }^{54}$ and equilibrate further to allow for relaxation of alkyl tails. A typical snapshot of an equilibrated configuration is shown in Fig. 2. The headgroups are adsorbed to the surface via the strong $\mathrm{Au}-\mathrm{S}$ interaction. The hydrocarbon tails form a soft corona. We find that the maximum coverage on $\mathrm{Au}_{147}\left(d_{c}=1.8 \mathrm{~nm}\right), \mathrm{Au}_{561}\left(d_{c}=2.7 \mathrm{~nm}\right)$, and $\mathrm{Au}_{1415}\left(d_{c}=3.7 \mathrm{~nm}\right)$ is 58,136, and 242 alkyl thiol molecules, respectively, in good agreement with experiments. ${ }^{55}$

\section{PAIR INTERACTIONS}

In this section, our results on pair interactions between capped gold NCs are presented. As a typical result, the PMF between two $\mathrm{Au}_{147}\left(\mathrm{SC}_{4}\right)_{58}$ clusters is shown in Fig. 3. The

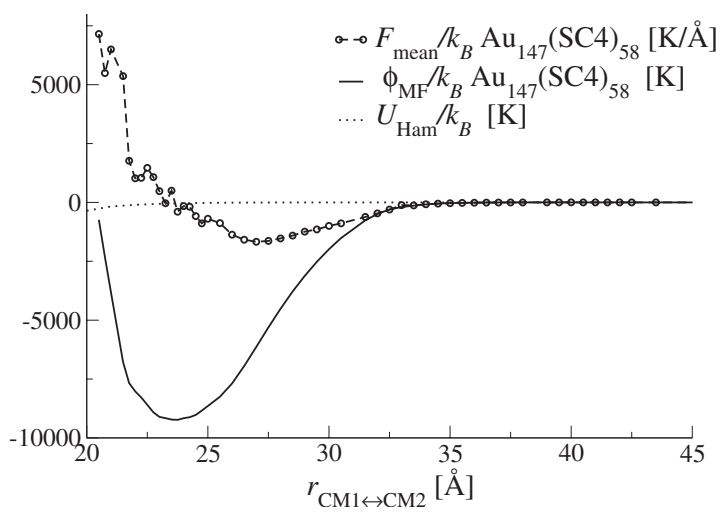

FIG. 3. Mean force $F_{\text {mean }}$, PMF $\phi_{\mathrm{MF}}$ and Hamaker interaction energy $U_{\text {Ham }}$ as functions of the center of mass separation $r$ between two $\mathrm{Au}_{147} \mathrm{NCs}$ $\left(d_{c}=1.8 \mathrm{~nm}\right)$ capped by 58 butane-thiol molecules $\left(\mathrm{SC}_{4}\right)$ each in vacuum, computed using constraint MD. The left end of the horizontal axis corresponds to the fusion distance $\left(2 r_{\max }\right)$ : twice the center to corner distance of a single NC. 


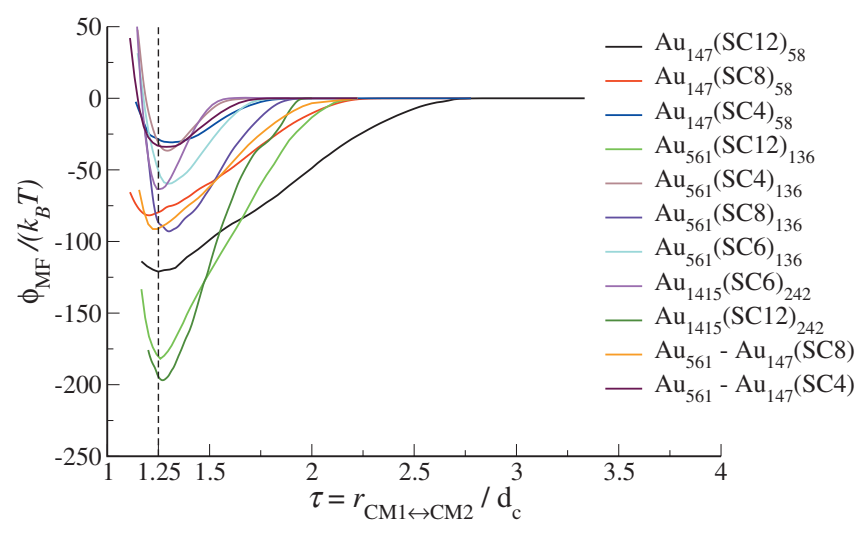

FIG. 4. PMF $\phi_{\mathrm{MF}}$ for different NC sizes and ligands as a function of center of mass separation $r$ divided by the NC core diameter $d_{c}$. This ratio is referred to as $\tau$ in the main text. The three $\mathrm{NCs}, \mathrm{Au}_{147}, \mathrm{Au}_{561}$, and $\mathrm{Au}_{1415}$, have core diameter $d_{c}=1.8,2.7$, and $3.7 \mathrm{~nm}$, respectively; capping molecules range from butanethiol $\mathrm{SC}_{4}(L \approx 0.5 \mathrm{~nm})$ to dodecanethiol $\mathrm{SC}_{12}$ $(L \approx 1.5 \mathrm{~nm})$. The vertical dashed line indicates $\tau=1.25$ and serves as guide to the eye.

left end of the horizontal axis is chosen as twice the center to corner distance $r_{\max }$. We will call $2 r_{\max }$ the fusion distance. Note that $2 r_{\max }>d_{c}$ because the NC is not perfectly spherical (for icosahedral NCs, $2 r_{\max } \approx 1.1 d_{c}$ ). The PMF has a strong repulsion at distances close to the fusion distance followed by a very deep well of $\approx 30 k_{B} T$. The attraction ranges a few angstroms beyond $2 r_{\text {max }}$ plus twice the ligand length (which is ca. $10 \AA$ here). The Hamaker interaction between the gold cores is negligible compared to the total interaction as found earlier. ${ }^{7,11,12,23}$ As the vdW interactions decay fast, the PMF is dominated by interactions between the entities that are in closest proximity, which are in our case the capping molecules.

We summarize the computed PMFs for different NC cores and ligands in Fig. 4. In this figure, we plot $\phi_{\mathrm{MF}}$ as function of the scaled distance $\tau=r / d_{c}$. The potential minima of all PMFs lie, surprisingly, in the narrow range $\tau$ $=1.25 \pm 0.04$ and do not depend significantly on the length $L$ of the capping molecule. Note that the ratio $\lambda=2 L / d_{c}$ varies in this figure between 0.27 and 1.67, which is an order of magnitude larger than the variation of $\tau$. The separation between NC surfaces at the equilibrium distance is for most systems less than the length of a single capping molecule in a stretched conformation. Figure 2(b) shows that this is possible because of the flexibility of aliphatic tails: The capping molecules located close to the NC-NC axis point in the direction perpendicular to this. Therefore, the alkyl tail length is not necessarily a restriction for $\mathrm{NC}$ spacing. We will come back to this issue in Sec. V where we will show that $\tau$ $\approx 1.25$ is the distance at which the ligands of any length are packed very efficiently in the space between two NCs. We extended the evidence for universal scaling by computing the PMF between a pair of NCs with different sizes $d_{c}^{(1)}$ and $d_{c}^{(2)}$ capped by the same ligand: $\mathrm{Au}_{561}\left(\mathrm{SC}_{n}\right)_{136}-\mathrm{Au}_{147}\left(\mathrm{SC}_{n}\right)_{58}$, with $n=4$ and 8 . For such a dimer, it is natural to define an effective NC diameter $\bar{d}_{c}=(1 / 2)\left(d_{c}^{(1)}+d_{c}^{(2)}\right)$. As can be seen from Fig. 4, the location of the minimum again does not change with increasing chain length, and still the ratio is $\tau$ $\approx 1.25$.

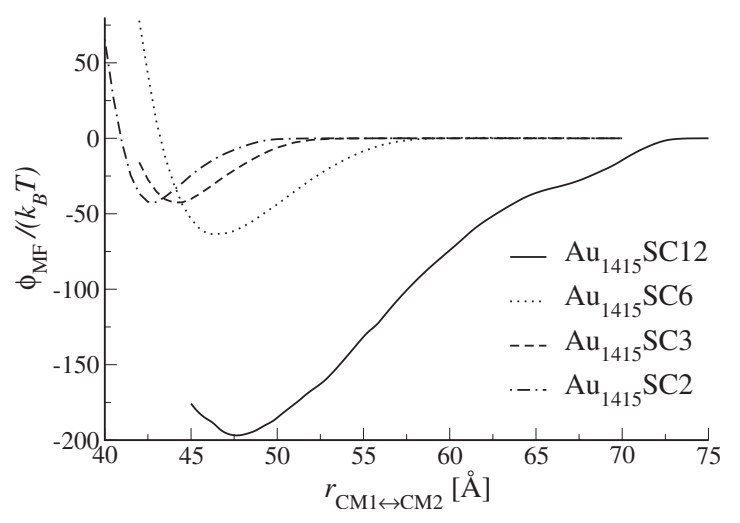

FIG. 5. PMF $\phi_{\mathrm{MF}}$ as a function of center of mass separation $r$ for $\mathrm{Au}_{1415}$ $\left(d_{c}=3.7 \mathrm{~nm}\right)$ capped by $\mathrm{SC}_{2}, \mathrm{SC}_{3}, \mathrm{SC}_{6}$, and $\mathrm{SC}_{12}$.

This scaling result allowed us to formulate a golden rule: ${ }^{23}$ "NC superstructures with $\tau$ close to 1.25 have an enhanced robustness and thermodynamic stability due to the large energetic contribution of the capping molecules." NC separations with $\tau \approx 1.25$ are expected when capped NCs self-assemble into one-dimensional (1D) structures such as rings or chains. Distances between adjacent capped NCs that are significantly smaller than twice the average surfactant length are often observed experimentally for thin NC films, with the ratio $\tau$ between 1.2 and $1.33 .^{4,10,56-61}$ In Ref. 10, a monolayer of $\mathrm{Au}$ NCs with $d_{c}=6 \mathrm{~nm}$ capped by $\mathrm{SC}_{12}$ was shown to form a self-supported membrane when dropcasted on a substrate with an up to $2 \mu \mathrm{m}$ large hole. The spacing between adjacent NCs was found to be $1.4 \mathrm{~nm}$, which is less than a single surfactant chain length. This corresponds to $\tau$ $=1.23$, and this is an excellent example of a robust monolayer obeying the golden rule. In 3D structures on the other hand, the typical distance between adjacent NCs depends on the ligand length due to the limited available volume, and our golden rule does not apply. ${ }^{5,62}$

The universal equilibrium distance of $1.25 d_{c}$ only makes sense if the ligand is sufficiently long; if $L<1.25 d_{c}-d_{c} / 2$, then the capping layers are not in contact when the NCs are at distance $r=1.25 d_{c}$. We computed the PMFs for a larger $\mathrm{NC} \mathrm{Au}_{1415}\left(d_{c}=3.7 \mathrm{~nm}\right)$ capped by very short ligands $\mathrm{SC}_{2}$ and $\mathrm{SC}_{3}$ and compared them to the ones for longer $\mathrm{SC}_{6}$ and $\mathrm{SC}_{12}$ capping molecules, see Fig. 5. Both $\mathrm{SC}_{2}$ and $\mathrm{SC}_{3}$ are significantly shorter than $1.25 d_{c}-d_{c} / 2=4.63 \AA$, and the spacing between the two NCs at the equilibrium distance for these short thiols equals 5.0 and $6.8 \AA$, respectively, which is in both cases approximately twice the ligand length. In contrast with this, the equilibrium distances for $\mathrm{SC}_{6}$ and $\mathrm{SC}_{12}$ (46.0 and $47.5 \AA$, respectively) are close to $1.25 d_{c}=46.9 \AA$ and show a much weaker dependence on the chain length.

Figure 4 indicates that the PMF well depth is mainly determined by the ligand length alone, and not by the $\mathrm{NC}$ size (and thus not by the number of capping molecules at full coverage). The only exception is $\mathrm{Au}_{147}\left(\mathrm{SC}_{12}\right)_{58}$. The values $U_{\min }$ of the potential well depth for different ligands $\mathrm{SC}_{n}$ can be fitted to the empirical formula $U_{\min } / k_{B} T=-u_{0}(n+1)^{2}$, and the parameter $u_{0} \approx 1.15$ is in first approximation NC size independent, see Fig. 6. In particular, the effective attraction scales quadratically with the number of beads in the capping 


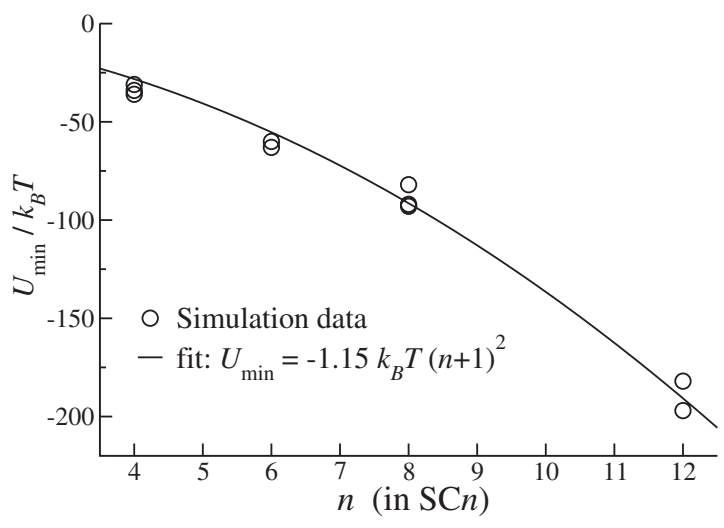

FIG. 6. Potential well depths $U_{\text {min }}$ from Fig. 4 plotted against the number $n$ of carbon atoms in the ligand tail. The value for $\mathrm{Au}_{147}\left(\mathrm{SC}_{12}\right)_{58}$ is omitted.

molecule. We have shown earlier that the prefactor $u_{0}$ decreases significantly with increasing temperature. ${ }^{23}$ A close comparison of the PMFs between two NCs of different size capped by the same surfactant yields that for larger NCs, the repulsion is steeper compared to smaller ones, and the potential well is deeper and more narrow. ${ }^{23}$ These observations can be explained by the smaller surface curvature for the larger NC, yielding a smaller angle between adjacent surfactants and therefore a denser capping layer. For the same reason, the PMF well depth for $\mathrm{Au}_{147}\left(\mathrm{SC}_{12}\right)_{58}$ is much lower than for $\mathrm{Au}_{561}\left(\mathrm{SC}_{12}\right)_{136}$ or $\mathrm{Au}_{1415}\left(\mathrm{SC}_{12}\right)_{242}$, see Fig. 4.

\section{A. Parameterization of effective NC pair interactions}

To make the computed pair interactions applicable in further studies, it is desirable to develop an interaction model for capped $\mathrm{Au}$ NCs that depends on the core diameter $d_{c}$ and the number of carbon atoms in the alkyl tail $n$. One can estimate the ligand length $L$ by applying the commonly used empirical formula $L \approx(n+1) \times 1.2 \AA$. $^{5,7}$ In this section, we present a potential form that captures the basic properties of the effective interactions computed in Sec. III. Note that we do not try to make a collective fit of all PMFs. We only consider here the case that the ligand is not too short: $L \geq\left(1.25 d_{c}-d_{c}\right) / 2$ and the temperature is $T=300 \mathrm{~K}$.

We suggest the following potential form for the twobody interactions:

$$
\phi_{\mathrm{MF}}^{\text {bbody }}(r)=f_{\mathrm{sm}}(r)\left(a\left(r-r_{\mathrm{cut}}\right)+b \exp \left\{-c\left(r-r_{\mathrm{min}}\right)\right\}\right),
$$

where $r_{\text {cut }}=1.2 d_{c}+2 L$ is the cutoff radius, the parameter $c$ determines the repulsion steepness, the parameters $a$ and $b$ can be expressed in terms of $c$, the location $r_{\min }=1.25 d_{c}$, and the value $U_{\text {min }}$ of the potential minimum by

$$
a=\frac{U_{\text {min }}}{r_{\text {min }}-r_{\text {cut }}+1 / c}, \quad b=\frac{a}{c}=\frac{U_{\text {min }}}{\left(r_{\min }-r_{\text {cut }}\right) c+1} .
$$

The smoothing cutoff function is given by

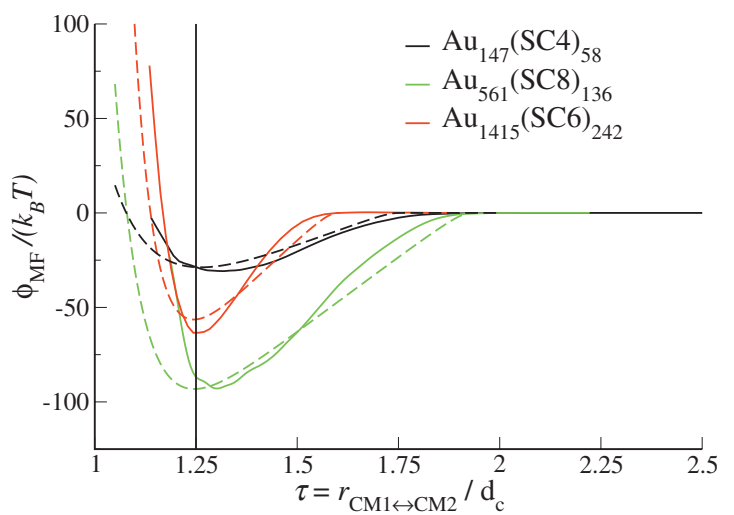

FIG. 7. Some computed two-body PMFs from Fig. 4 (solid lines) and the potential Eq. (7) with the parameters corresponding to each system (dashed lines). As in Fig. 4, the scaled distance $\tau$ is used on the horizontal axis and the vertical line indicates $\tau=1.25$.

$$
f_{\mathrm{sm}}(r)= \begin{cases}\exp \left(0.05 \frac{d_{c}\left(r_{\text {min }}-r\right)}{\left(r-r_{\text {cut }}\right)\left(r_{\text {min }}-r_{\text {cut }}\right)}\right) & r<r_{\text {cut }} \\ 0 & r \geq r_{\text {cut }} .\end{cases}
$$

When choosing $c=0.55 \AA^{-1}, r_{\min }=1.25 d_{c}$, and $U_{\min } / k_{B} T$ $=-1.15(n+1)^{2}$, the potential Eq. (7) reproduces the following properties observed in Sec. III:

- The equilibrium distance is for any ligand at $r_{\min }$ $=1.25 d_{c}$.

- The interaction ranges a few angstroms beyond $r_{\mathrm{ovrl}}^{2 b}$ $=d_{c}+2 L$, which is the $\mathrm{NC}$ diameter plus twice the ligand length; for $r>r_{\text {ovrl }}^{2 b}$ the PMF smoothly approaches 0 .

- The potential well depth is $U_{\min } / k_{B} T=-1.15(n+1)^{2}$.

- The attractive part of the two-body PMF is almost linear.

- The repulsion is steep in the range $1.1 d_{c}<r<1.25 d_{c}$.

Moreover, both the potential and its derivative are continuous at the cutoff owing to the smoothing function $f_{\mathrm{sm}}$. Three typical PMFs from Fig. 4 with different NC cores and ligands are compared to the potentials from Eq. (7) in Fig. 7. The agreement is reasonable when taking into account that we did not perform a collective fit. It can be seen on the example of $\mathrm{Au}_{561}\left(\mathrm{SC}_{8}\right)$ that the differences are mainly due to the small deviations of the imposed values for $r_{\min }$ and $U_{\min }$ from the computed ones.

\section{THREE-BODY EFFECTS}

In the previous section, we have studied interactions between pairs of capped NCs. The crucial question is in which cases are these pair potentials sufficient to reasonably model large NC superstructures. We found that, surprisingly, the equilibrium distance of $\mathrm{NC}$ dimers is independent from the ligand length $L$ and is $\approx 1.25 d_{c}$, where $d_{c}$ is the NC core diameter (see Fig. 4). By contrast, experiments show that the spacing between NCs in a 3D-superlattice systematically in- 


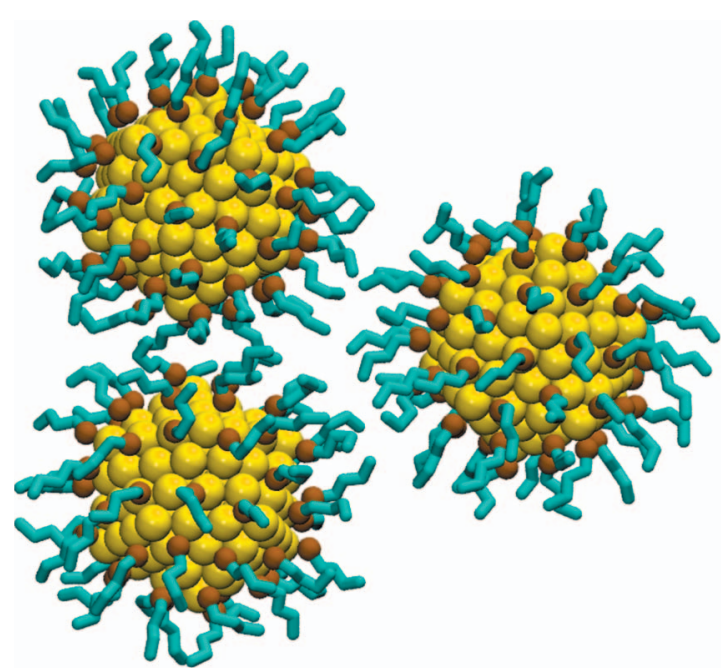

FIG. 8. A simulation snapshot of a $\mathrm{Au}_{147}\left(\mathrm{SC}_{4}\right)_{58}$ triplet with $R_{12}=24.5 \AA$ and $r_{3 C}=27 \AA$. Representations are the same as in Fig. 2.

creases with increasing length of the capping molecules at constant core diameter. ${ }^{5,62,63}$ This indicates that pair interactions alone may not be appropriate to describe 3D superstructures built from NCs with long capping molecules. On the other hand, pair potentials are sufficient to describe the energy of 1D superstructures (chains or rings) of capped NCs. In a similar fashion, a study of three-body interactions would allow us to apply the computed effective interactions to simulate 2D NC superstructures (monolayers). We are not aware of systematic experimental studies of monolayers made from $\mathrm{Au} \mathrm{NCs}$ with constant core size and varying ligand length. In Ref. 64 it was shown that the spacing between $5.8 \mathrm{~nm} \mathrm{Ag}_{2} \mathrm{~S}$ NCs increases nonlinearly with $L$, and the spacing was almost the same for $\mathrm{SC}_{12}$ and $\mathrm{SC}_{14}$. This indicates that three-body effects may determine the spacing between NCs in a nontrivial way.

We consider two systems with the same $\mathrm{NC}\left(d_{c}\right.$ $=18 \AA$ ) capped by butane and octanethiol: $\mathrm{Au}_{147}\left(\mathrm{SC}_{4}\right)_{58}$ and $\mathrm{Au}_{147}\left(\mathrm{SC}_{8}\right)_{58}$. A typical simulation snapshot is shown in Fig. 8. The geometric properties of the two systems are characterized by the following distances, which are summarized in Table III:

- Ligand length $L$.

- NC core diameter $d_{c}=18 \AA$.

- Interpenetration distance $r_{\mathrm{ovrl}}^{2 b}=d_{c}+2 L$ between two NC centers, which is the center-to-center distance for a $\mathrm{NC}$ pair at which the two capping layers touch each other.

TABLE III. Geometric data of the two NC systems described in the text. All distances are reported in angstrom.

\begin{tabular}{lcccc}
\hline \hline & $L$ & $r_{\text {ovrl }}^{2 b}$ & $r_{\text {ovrl }}^{3 b}$ & $r_{\text {eq }}^{2 \text { body }}$ \\
\hline Formula & & $d_{c}+2 L$ & $\frac{\sqrt{3}}{2} r_{\text {ovrl }}^{2 b}$ & \\
$\mathrm{Au}_{147}\left(\mathrm{SC}_{4}\right)_{58}$ & 4.8 & 27.6 & 23.9 & 23.7 \\
$\mathrm{Au}_{147}\left(\mathrm{SC}_{8}\right)_{58}$ & 9.6 & 37.2 & 32.2 & 21.6 \\
\hline \hline
\end{tabular}

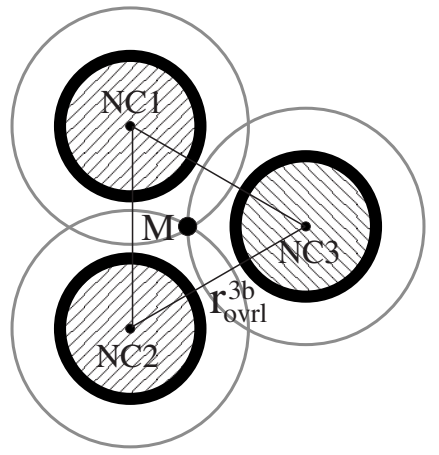

FIG. 9. Representation of the triplet overlap distance $r_{\text {ovrl }}^{3 b}$. The capping layer boundaries of the three NCs, represented by gray lines, intersect in the midpoint $M$ of the equilateral triangle with corners in NC1, NC2, and NC3.

- Triplet interpenetration distance $r_{\mathrm{ovrl}}^{3 b}=\left(\frac{d_{c}}{2}+L\right) \sqrt{3}$ between two NC centers: If three NCs are arranged in a equilateral triangle, this would be the maximum side length at which the three capping layers overlap in the triangle midpoint (see Fig. 9).

- Equilibrium distance of the two-body PMF $r_{\mathrm{eq}}^{\text {2body }}$, taken from Fig. 4.

- Equilibrium distance of the effective two-body PMF in the three-body system, $r_{\mathrm{eq}}^{\mathrm{eff}}$.

The $\mathrm{SC}_{4}$ ligand is relatively short $(\approx 5 \AA)$, and the interpenetration range at the well depth of the associated PMF is slightly smaller than one capping molecule. The second ligand $\left(\mathrm{SC}_{8}\right)$ is two times longer than $\mathrm{SC}_{4}$ and has a very large interpenetration range of more than 1.5 capping molecules, see Fig. 4. For the $\mathrm{SC}_{4}$ ligand, we chose $R_{12}$ separations of 23.5 and $24.5 \AA$, which are both close to the dimer equilibrium distance $r_{\text {eq }}^{\text {body }}$. For the longer $\mathrm{SC}_{8}$ ligand, we computed the effective pair interaction $\phi_{\mathrm{MF}}^{\text {eff }}$ for $R_{12}=23.5$, 24.5, 26.0, and $30.0 \AA$.

The calculated effective forces $F_{\text {eff }}$ and pair interactions $\phi_{\mathrm{MF}}^{\mathrm{eff}}$ for the two systems are shown in Figs. 10 and 11, respectively. If no three-body effects were present, $\phi_{\mathrm{MF}}^{\text {eff }}$ would be identical to the two-body PMF $\phi_{\mathrm{MF}}^{\text {2body }}$ from Sec. III. The difference between $\phi_{\mathrm{MF}}^{\text {eff }}$ and $\phi_{\mathrm{MF}}^{2 \text { body }}$ is in all cases very small for $r>r_{\text {ovrl }}^{2 b}$, i.e., when the capping layer of NC3 does not overlap with the other two capping layers. For smaller $r_{13}$ distances, the three-body interaction becomes increasingly repulsive. In all cases, the equilibrium distance shifts toward larger separations.

In a $\mathrm{Au}_{147}\left(\mathrm{SC}_{4}\right)_{58}$ triplet, the effective interaction between NC pairs becomes $20 \%$ less attractive compared to the two-body case. The location of the $\phi_{\mathrm{MF}}^{\mathrm{eff}}$ well depth decreases by $1 \AA$ ( $4.2 \%)$ compared to $\phi_{\mathrm{MF}}^{\text {2body }}$. The effective interactions $\phi_{\mathrm{MF}}^{\text {eff }}$ computed for two different $R_{12}$ separations lie within statistical accuracy. When a $\mathrm{Au}_{147}\left(\mathrm{SC}_{4}\right)_{58}$ triplet is arranged in an equilateral triangle with side length equal to $r_{\mathrm{eq}}^{\text {eff }}$ $\approx 24.7 \AA$, the distance from each NC center to the midpoint of the triangle is $r_{\mathrm{eq}}^{\text {eff }} / \sqrt{3}=14.3 \AA$. This is slightly larger than the distance $d_{c} / 2+L$ from a NC center to the capping layer layer boundary, so that there the three capping layers only overlap pairwise. On the other hand, in a triangle with side equal to $r_{\mathrm{eq}}^{\text {body }} \approx 23.7 \AA$, the distance to the triangle midpoint 


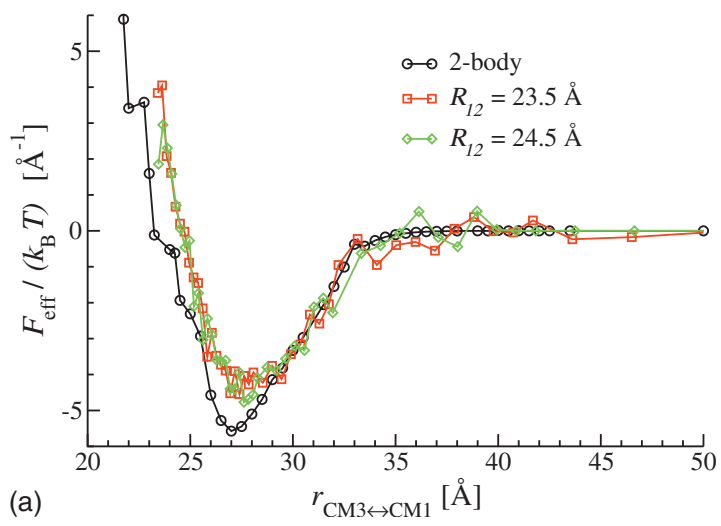

(a)

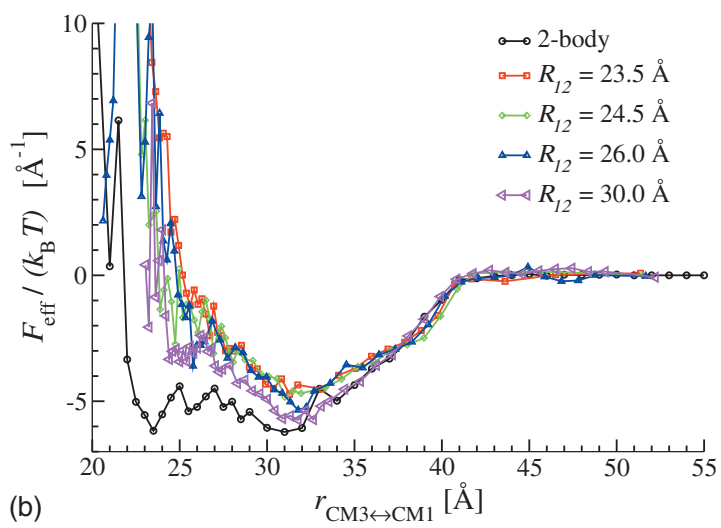

FIG. 10. Effective force $F_{\text {eff }}$ in a three-NC system from Fig. 1 as a function of $\mathrm{NC}$ distance $r_{13}$ for (a) $\mathrm{SC}_{4}$ and (b) $\mathrm{SC}_{8}$ triplets.

would be $13.7 \AA$, which is slightly smaller than $d_{c} / 2+L$. This would allow an overlap of the three capping layers. Thus, for the short ligand the shift of the equilibrium distance can be rationalized as avoiding a triplet overlap.

For a $\mathrm{Au}_{147}\left(\mathrm{SC}_{8}\right)_{58}$ system, the effective interactions corresponding to $R_{12}=23.5,24.5$, and $26.0 \AA$ are very similar. As expected, $\phi_{\mathrm{MF}}^{\text {eff }}$ corresponding to $R_{12}=30.0 \AA$ lies between the one for $R_{12}=26.0 \AA$ and the two-body PMF $\phi_{\mathrm{MF}}^{2 \mathrm{body}}$, as the three-body effects become weaker for increasing separation $R_{12}$ between $\mathrm{NC} 1$ and $\mathrm{NC} 2$. The location of the $\phi_{\mathrm{MF}}^{\text {eff }}$ well depth is decreased by $3 \AA$ (14\%) compared to $\phi_{\mathrm{MF}}^{2 \text { body }}$. The effective pair interaction $\phi_{\mathrm{MF}}^{\text {eff }}$ is up to $\approx 40 \%$ less attractive than $\phi_{\mathrm{MF}}^{2 \text { body }}$. This may have a strong consequence

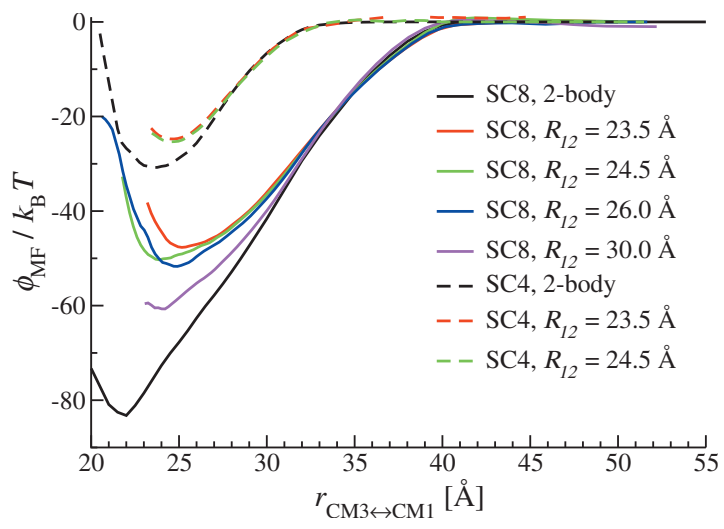

FIG. 11. Effective pair potential $\phi_{\mathrm{MF}}^{\mathrm{eff}}$ in a three-NC system from Fig. 1 as a function of $\mathrm{NC}$ distance $r_{13}$ in $\mathrm{Au}_{147}\left(\mathrm{SC}_{4}\right)_{58}$ and $\mathrm{Au}_{147}\left(\mathrm{SC}_{8}\right)_{58}$ triplets. The two-body interactions are taken from Fig. 4.

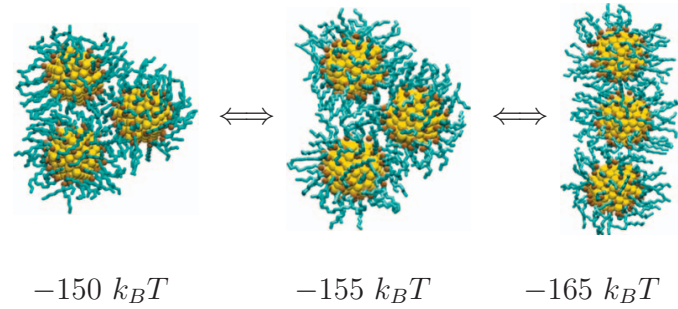

FIG. 12. Three different configurations of a $\mathrm{Au}_{147}\left(\mathrm{SC}_{8}\right)_{58}$ triplet: equilateral triangle, chain, and an intermediate configuration. The total interaction energies of the clusters are computed using Fig. 11.

for the formation of $\mathrm{Au}_{147}\left(\mathrm{SC}_{8}\right)_{58}$ aggregates at the air-water interface. In the pioneering study of Heath et al. it was observed that NCs capped by long ligands organize into linear structures at the air-water interface at low surface pressures, while foamlike structures form at higher surface pressures. ${ }^{3}$ The authors of this study already speculated that effective three-NC interactions are responsible for such behavior. Our work provides direct evidence for this speculation. This can be seen as follows. Consider two possible configurations for a triplet of $\mathrm{Au}_{147}\left(\mathrm{SC}_{8}\right)_{58}$ clusters: Three in a row and equilateral triangle, each with energetically most favorable distances, see Fig. 12. In the first case, three-body interactions are absent, and the total energy of this configuration is twice the well depth of $\phi_{\mathrm{MF}}^{\text {2body }}$, which is $U_{\text {chain }} / k_{B} T \approx-165$. In the second case, the energy of the triplet is three times the well depth of $\phi_{\mathrm{MF}}^{\text {eff }}$. From the three PMFs corresponding to $R_{12}$ $=23.5,24.5$, and $26.0 \AA$, we can make an estimate $U_{\text {triangle }} / k_{B} T=-150 \pm 5$. Once a triangle is formed, it can easily "straighten," as shown in Fig. 12. To see this, consider a triangle with $R_{12}=30 \AA$, and $r_{13}=r_{23}=24 \AA$, which is a possible intermediate configuration between an equilateral triangle and a chain. From the effective interactions in Fig. 11, we can estimate the energy of this configuration as $\approx$ $-155 k_{B} T$ (assuming a NC1-NC2 contribution of $35 k_{B} T$ ). Therefore, the linear arrangement of the triplet is energetically preferred over the triangle for long capping molecules. This is an interesting example of a spontaneous anisotropic assembly of isotropic entities.

Interestingly, $r_{\mathrm{eq}}^{\text {eff }}$ is the same for the two ligands despite the difference in length by $5 \AA$. The question whether this agreement is coincidental or systematic cannot be answered with the available data; further investigation is necessary using both experiments and simulations. On one hand, the separation between $\mathrm{SC}_{4}$-capped $\mathrm{NCs}$ can be explained in terms of the avoiding of a triple overlap (see above). Independently from this argument, we will show in Sec. V that the three-body equilibrium distance for $\mathrm{SC}_{8}$-capped $\mathrm{NCs}$ can be understood as the densest possible packing of ligand chains. By contrast, a "ligand-independent" three-body equilibrium distance would be a possible continuation of the universal scaling of the two-body interactions found in Sec. III.

\section{A. Parametrization of triplet interactions}

In Sec. IV we have seen that three-body effects are not very large for a relatively short ligand $2 L<0.5 d_{c}$. We feel that in this range they can either be neglected completely or considered implicitly by making the two-body interactions 
from Sec. III A slightly less attractive. However, three-body effects for a long ligand are much larger than for a short one. If one either neglects them or replaces them by an effective pair interaction, it is expected that several important properties of the system will not be reproduced correctly. In this section, we present a model for effective interactions in a $\mathrm{Au}_{147}\left(\mathrm{SC}_{8}\right)_{58}$ triplet that captures our main findings from Sec. IV on this system.

We propose the following correction term for each of the pair interactions $\phi_{\mathrm{MF}}$ :

$$
\begin{aligned}
\phi_{\mathrm{MF}}^{\mathrm{eff}}\left(r_{i j}\right)-\phi_{\mathrm{MF}}^{2 \mathrm{body}}\left(r_{i j}\right) & \\
= & \kappa\left(r_{\mathrm{ovrl}}^{2 b}-r_{i j}\right)^{3}\left(r_{\mathrm{ovrl}}^{2 b}-r_{i k}\right)^{2}\left(r_{\mathrm{ovrl}}^{2 b}-r_{j k}\right)^{2} \\
& \Theta\left(r_{\mathrm{ovrl}}^{2 b}-r_{i j}\right) \Theta\left(r_{\mathrm{ovrl}}^{2 b}-r_{i k}\right) \Theta\left(r_{\mathrm{ovrl}}^{2 b}-r_{j k}\right),
\end{aligned}
$$

where the indices $i, j, k$ run over 1,2 , and 3. The Heaviside step function $\Theta$ is defined as

$$
\Theta(x)=\left\{\begin{array}{ll}
1 & x>0 \\
0 & x<0
\end{array},\right.
$$

and the parameter $\kappa / k_{B} T \approx 3 \times 10^{-7} \AA^{-7}$ for the $\mathrm{Au}_{147}\left(\mathrm{SC}_{8}\right)_{58}$ system that we studied in Sec. IV. This potential reproduces the following properties of the effective interaction in $\mathrm{Au}_{147}\left(\mathrm{SC}_{8}\right)_{58}$ triplet:

- The three-body correction vanishes if any distance in a triplet becomes larger than $r_{\mathrm{ovrl}}^{2 b}=d_{c}+2 L$, which is guaranteed by the $\Theta$-functions.

- The correction term becomes larger when each of the three distances becomes shorter.

- The equilibrium distance in an equilateral triangle increases by $4 \AA$; the potential well becomes $40 \%$ higher.

Moreover, the resulting potential and its derivatives are continuous. The distance $r_{i j}$ is given a stronger weight through the cubic power. Note that the correction term Eq. (8) should be added for each of the three sides of a triangle, and not one time per triplet as it is the case in several manybody models.

A typical potential energy surface for the $\mathrm{Au}_{147}\left(\mathrm{SC}_{8}\right)_{58}$ triplet computed using Eq. (7) for $\phi_{\mathrm{MF}}^{2 b o d y}$ and Eq. (8) for the three-body correction is shown in Fig. 13. Note that the three-body correction term not only weakens the attraction, but also strongly deforms the low-energy regions. The minimum-energy region is parallel to the $\mathrm{NC} 1-\mathrm{NC} 2$ pair when the three-body correction is added [see Fig. 13(a)], which is not the case without the correction term [see Fig. 13(b)].

\section{EQUILIBRIUM DISTANCE BY OPTIMAL PACKING OF LIGANDS}

In this section we will rationalize the scaling results for the equilibrium distance between capped NCs found in Secs. III and IV. The variables of interest are the center to center distance $r$ between a pair of NCs with diameter $d_{c}$ and ligand length $L$. Here, NC cores are considered as spheres of diameter $d_{c}$. It is convenient to introduce scaled variables $\tau$

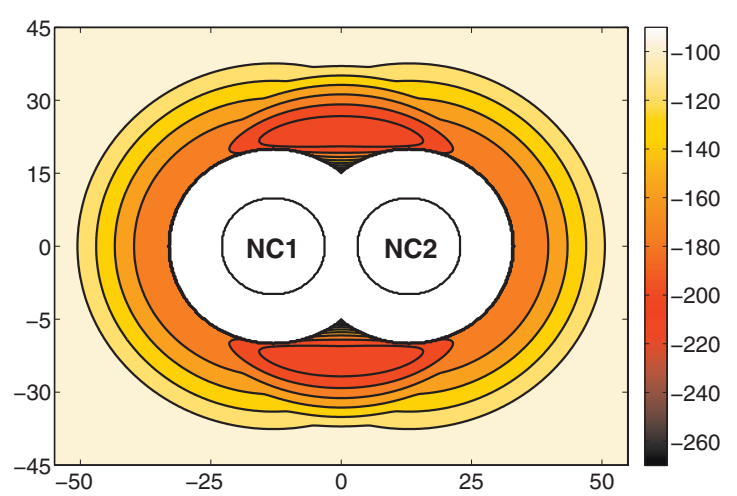

(a)

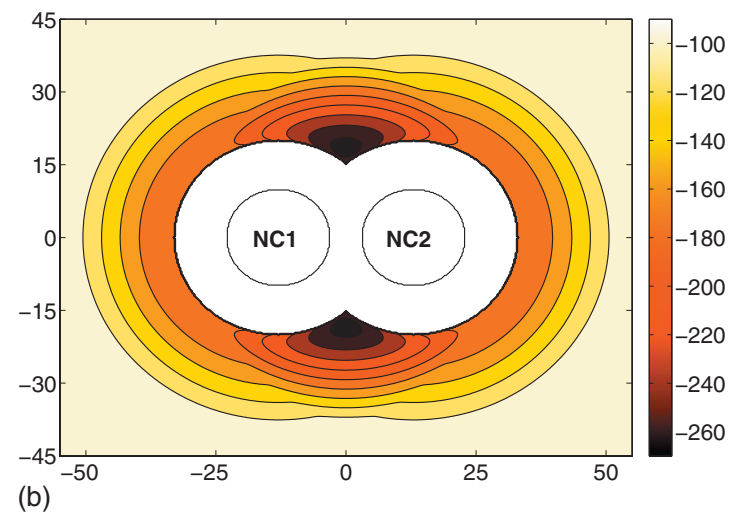

FIG. 13. Potential energy surface of a $\mathrm{Au}_{147}\left(\mathrm{SC}_{8}\right)_{58}$ triplet as a function of position of the center of the third NC. The distance between the first and the second $\mathrm{NC}\left(\mathrm{NC} 1\right.$ and $\mathrm{NC} 2$ ) is fixed at $R_{12}=26 \AA$. The energy in units of $k_{B} T$ is computed (a) using the pair potential Eq. (7) with the three-body correction of Eq. (8) and (b) using only the pair potential Eq. (7). The unit of distance is angstrom. The inner white circles represent the cores of NC1 and $\mathrm{NC} 2$; the white rings around them are the regions that are not accessible to the center of the third $\mathrm{NC}$ due to core-core overlap. These regions should not be confused with the capping layers.

$=r / d_{c}$ and $\lambda=2 L / d_{c}$. The goal of this section is to establish a relation for the equilibrium distance $\tau_{\mathrm{eq}}$ of the form $\tau_{\mathrm{eq}}$ $=\tau_{\mathrm{eq}}(\lambda)$.

The equilibrium distance between a pair of interacting capped NCs is the separation at which the total force on each $\mathrm{NC}$ vanishes. Given the strong vdW-attraction between capping layers in vacuum, the repulsive forces must also become very large at the equilibrium distance. This happens if one or more ligand molecules are overcompressed, as an alkane chain requires a certain minimum volume. If a capping molecule is confined into a too small volume, it exerts a large repulsive force. This observation suggests the following constituting equation for all packing models:

$$
V_{\text {lig }}\left(\tau_{\text {eq }}, \lambda\right)=V_{\text {avail }}\left(\tau_{\text {eq }}, \lambda\right),
$$

where $V_{\text {lig }}$ is the volume required for a certain set of ligand molecules, and $V_{\text {avail }}$ is the volume available to this set. This approach was originally introduced in Ref. 3 and elaborated further in Ref. 7. First, one has to specify a relevant set of ligands and the volume available to them. This task is highly nontrivial and not unambiguous. We will show in the sequel that this choice affects the prediction of a packing model, especially for $\lambda \geq 0.5$. Second, one has to formulate the two volumes in Eq. (10) as functions of $\tau$ and $\lambda$. This can be done 


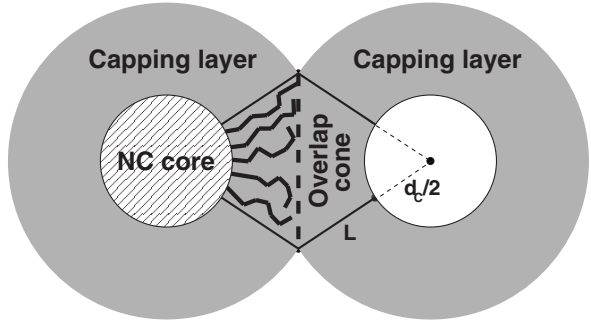

FIG. 14. A sketch illustrating the OCM (present work). By connecting the intersection plane (represented by the vertical dashed line) with a NC center, one obtains a cone. Truncation of this cone at the NC surface yields the overlap cone. It is then assumed that the ligands whose headgroups are adsorbed inside the overlap cone (represented by bold curved lines) lie completely inside the overlap cone.

either analytically or numerically. Finally, by solving Eq. (10) with respect to $\tau$, we obtain $\tau_{\text {eq }}(\lambda)$.

We start with general considerations concerning $V_{\text {lig. }}$. The number of ligand molecules on a metallic NC is limited by the repulsion between headgroups. Therefore, one introduces the ligand footprint $A_{0}$, which is the area on the NC surface occupied by one ligand. The volume $V_{1}^{0}$ of one single linear ligand is then $V_{1}^{0}=L A_{0}$. In an ideal situation (denoted by “ 0 "), the total number of ligands $N_{\text {lig }}^{0}$ on a fully capped NC is the surface area of the $\mathrm{NC}$ sphere divided by the footprint:

$$
N_{\text {lig }}^{0}=\frac{\pi d_{c}^{2}}{A_{0}} .
$$

The volume $V_{\text {lig }}^{0}$ of the ligands whose headgroups are adsorbed on a specific area $A_{\text {lig }}$ is then given by

$$
V_{\text {lig }}^{0}=\frac{A_{\text {lig }}}{A_{0}} V_{1}^{0}=A_{\text {lig }} L .
$$

To adapt the model to more general systems, one has to introduce the density parameter $\xi$, which is the product of the relative grafting density and relative ligand volume:

$$
\xi=\frac{N_{\text {lig }}}{N_{\text {lig }}^{0}} \frac{V_{1}}{V_{1}^{0}},
$$

where $N_{\text {lig }}$ is the actual number of capping molecules and $V_{1}$ is the actual ligand volume. Note that the inclusion of the parameter $\xi$ is essential for branched ligands, as $V_{1} / V_{1}^{0}>1$ in this case. The ligand volume $V_{\text {lig }}$ is related to the ideal ligand volume $V_{\text {lig }}^{0}$ by

$$
V_{\text {lig }}=\xi V_{\text {lig }}^{0} \text {. }
$$

Figure 2 shows the situation that ligands are relatively flexible, and that they are able to bend away from the "bottlenecks" between NC surfaces. The thiol headgroups, on the other hand, remain immobile as they are strongly adsorbed to the NC surface. We therefore propose the model sketched in Fig. 14. A pair of overlapping capping layers defines a circle in the intersection plane, which is the full contact area between the two capped NCs. By connecting this circle to each NC center, we obtain two overlap cones with volume $V_{\text {cone }}$ each. Obviously, a part of each cone with volume $V_{\text {cone }}^{\text {core }}$ belongs to the corresponding NC core. The remaining volume $V_{\text {cone }}-V_{\text {cone }}^{\text {core }}$ is then available to capping molecules. The area of the NC surface inside each cone is
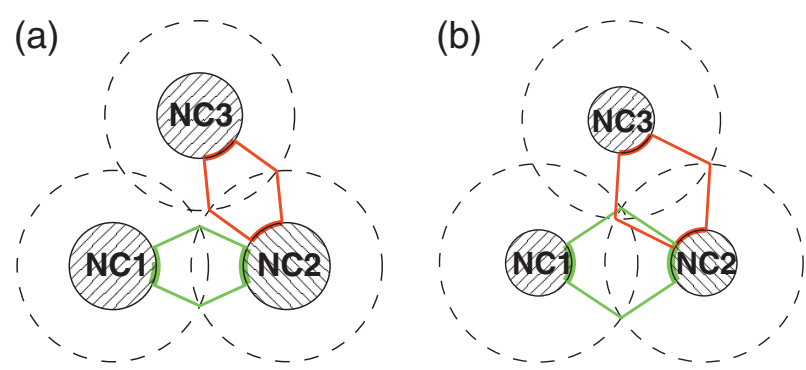

FIG. 15. Three-body effects in the OCM. The three NCs are represented by shaded circles, their capping layers by the corresponding dashed circles. The overlap cones between $\mathrm{NC} 1$ and $\mathrm{NC} 2$ and between $\mathrm{NC} 2$ and $\mathrm{NC} 3$ are represented by green and red lines, respectively. In (a), these two overlap cones do not intersect and they can be treated separately. In (b), the two overlap cones do intersect, so that both the intersection volume and the intersection NC surface area must be accounted for in Eq. (15). For clarity, the overlap cone between NC1 and NC3 as well as ligand molecules is not shown.

denoted by $A_{\text {cone }}^{\text {core }}$ Our main assumption is that the ligands adsorbed on this surface lie inside an overlap cone. We will refer to this assumption as OCM. Equation (10) then becomes

$$
V_{\text {lig }}=\xi A_{\text {cone }}^{\text {core }} L=V_{\text {cone }}-V_{\text {cone }}^{\text {core }}=V_{\text {avail }} .
$$

It is important to note that both sides of this equation depend on the ligand length and the distance between NCs. Moreover, due to symmetry the OCM does not distinguish whether the ligand molecules belonging to one $\mathrm{NC}$ stay completely inside the corresponding cone or lie partly in the other cone.

For a pair of NCs with equal size, the three terms in Eq. (15) can be calculated using the formulae for solids of revolution:

$$
\begin{aligned}
& \xi A_{\text {cone }}^{\text {core }} L=\frac{\pi d_{c}^{3}}{4} \lambda \xi\left(1-\frac{\tau}{1+\lambda}\right), \\
& V_{\text {cone }}=\frac{\pi d_{c}^{3}}{24} \tau\left((1+\lambda)^{2}-\tau^{2}\right), \\
& V_{\text {cone }}^{\text {core }}=\frac{\pi d_{c}^{3}}{12}\left(1-\frac{\tau}{1+\lambda}\right) .
\end{aligned}
$$

After dividing both sides by $\pi d_{c}^{3} / 8$, Eq. (15) becomes

$$
2 \xi \lambda\left(1-\frac{\tau}{1+\lambda}\right)=\frac{1}{3} \tau\left((1+\lambda)^{2}-\tau^{2}\right)-\frac{2}{3}+\frac{2}{3} \frac{\tau}{1+\lambda} .
$$

This is a cubic equation in $\tau$ and it always has a trivial solution $\tau=1+\lambda$, i.e., when the two capping layers just touch each other. Indeed, in this case both the cone volume $V_{\text {cone }}$ and the corresponding surface area $A_{\text {cone }}^{\text {core }}$ vanish. The relevant solution is

$$
\tau_{\mathrm{eq}}=-\frac{1+\lambda}{2}+\sqrt{\left(\frac{1+\lambda}{2}\right)^{2}+\frac{6 \xi \lambda+2}{1+\lambda}},
$$

while the third root of Eq. (17) is negative.

An important feature of the OCM is that it can account for many-body effects. When a capping layer of $\mathrm{NC1}$ overlaps with capping layers of two other NCs, it may occur that the two overlap cones on $\mathrm{NC} 1$ intersect as in Fig. 15. In this 


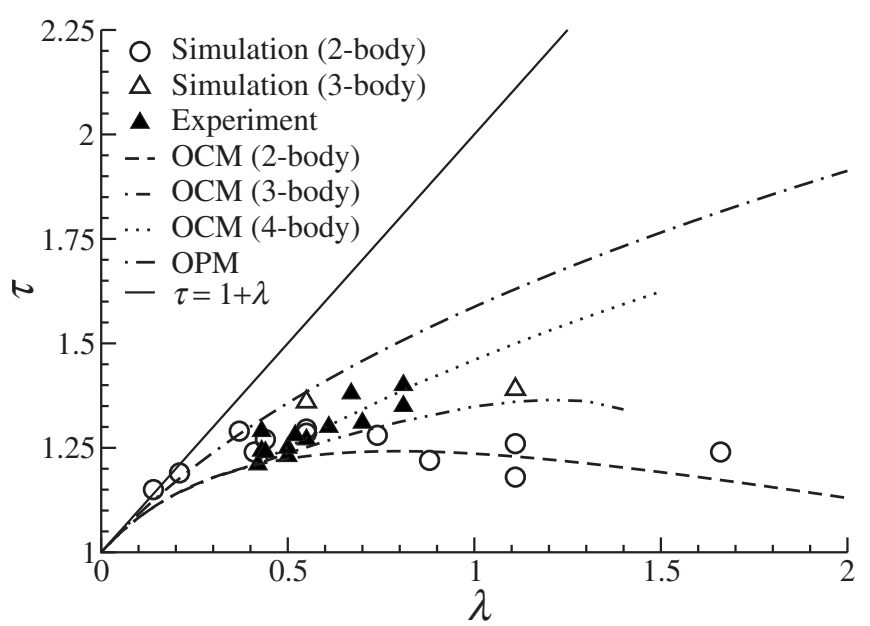

FIG. 16. Scaled equilibrium distance $\tau$ vs scaled ligand length $\lambda$. Results of our two-body (Sec. III) and three-body (Sec. IV) simulations together with experimental data from Refs. 2, 10, 56-59, 61, and 64 are compared with predictions of OCM (present work) and OPM (Ref. 7). The OCM data for two NCs were calculated using Eq. (18); the OCM data for three and four NCs were obtained by solving Eq. (15) numerically. The OPM data were calculated using Eq. (21). When the capping layers do not overlap, $\tau=1+\lambda$.

case, some ligands on $\mathrm{NC1}$ lie in two overlap cones simultaneously. Four-body effects arise in the same manner when four NCs are arranged in a tetrahedron. If one now wishes to express the volumes on both sides of Eq. (15) by combining expressions of Eq. (16), one has to subtract the doublecounted intersection volume of the two cones on the right side and the double-counted surface area on the left side of Eq. (17). Unlike the two-NC case, we were not able to calculate the corresponding integrals analytically. We solved Eq. (15) numerically for a NC triplet arranged in an equilateral triangle and for four NCs arranged in a regular tetrahedron. This numerical solution is shown in Fig. 16. The solution is identical to Eq. (18) for $\lambda \leq 0.39$, and then the aforementioned intersection of overlap cones occurs. For a tetrahedron, it is useful to consider the case when the ligands fill the whole space between NC cores (which holds for $\lambda$ $\geq 0.54$ ). The solution of Eq. (15) is then

$$
\tau_{\text {eq }}=\sqrt[3]{\eta_{\text {tetr }}(1+3 \xi \lambda)},
$$

where $\eta_{\text {tetr }} \approx 0.780$ is the atomic packing factor of a tetrahedron. Note that Eq. (19) is also a good (lower) approximation for $\tau_{\mathrm{eq}}$ in the interval $0.39<\lambda<0.54$ with the maximum deviation from the exact solution being less than 0.01 .

The optimal packing model (OPM) of Landman and Luedtke $^{7}$ makes different assumptions regarding the flexibility of ligand tails. First, the ligand cone is introduced: A cone with the vertex in the NC center through the ligand footprint. Second, the OPM assumes that in a dense 3D many-body superlattice the tail of a ligand molecule occupies some volume mainly inside this cone. Third, the ligand lying on the $\mathrm{NC}-\mathrm{NC}$ line is considered. Its ligand cone is truncated between the NC surface and the intersection plane of the two capping layers. Finally, the OPM postulates that the optimal packing is achieved when the volume of this truncated cone equals the volume $V_{1}$ of a single ligand. Thus, the OPM considers the central part of the contact region between two
NCs and makes a prediction for its density, whereas the OCM models the density of the entire contact region. In our notation, we can apply Eq. (10) to a part of the contact zone, resulting in

$$
V_{\text {lig }}=V_{1}=\xi A_{0} L=\frac{d_{c}}{6} A_{0}\left(\tau^{3}-1\right)=V_{\text {avail }},
$$

which is identical to Eqs. (1) and (2) of Ref. 7. The right part of this equation is the volume of the aforementioned truncated ligand cone. The only real solution of Eq. (20) is ${ }^{7}$

$$
\tau_{\text {eq }}=\sqrt[3]{1+3 \xi \lambda}
$$

It should be noted that OPM reproduces very well experimental data on 3D-superlattices of capped Au NCs from Ref. 5. It is not clear, however, whether it is applicable to monolayers or small clusters of NCs. Coincidentally, Eq. (21) is identical to Eq. (19) with atomic packing factor of $\eta=1$.

In Fig. 16, we plotted our results from Secs. III and IV together with available experimental data on monolayers of capped gold and silver NCs, and we compare them to predictions of OCM and OPM. For both models, we consider the generic case $\xi=1$. Both packing models predict equilibrium distances between NCs significantly smaller than $1+\lambda$, implying a large interpenetration of capping layers. Consider first the two-body OCM: $\tau_{\text {eq }}$ increases up to $\approx 1.25$ until $\lambda$ $\approx 0.75$ and then very slowly decreases. In particular, in the region $0.4 \leq \lambda \leq 1.5$ the value of $\tau_{\text {eq }}$ remains in the very narrow interval $1.2<\tau<1.25$. This explains the golden rule $(\tau \approx 1.25)$ that we established in Sec. III. The values of $\tau_{\mathrm{eq}}$ predicted by the three-body OCM become significantly higher than the ones predicted by the two-body OCM for $\lambda$ $>0.65$, although they differ already for $\lambda>0.39$. For a threebody system, the OCM predicts a systematic increase in $\tau_{\text {eq }}$ up to $\lambda \approx 1.3$. The four-body effects are even more pronounced. Overall, Fig. 16 demonstrates a systematic progression from two-body, three-body over four-body systems (OCM) to 3D many-body systems (OPM).

The OCM reproduces both experimental and simulation data very well in the region $0.35<\lambda<0.6$. For $\lambda>0.65$, the difference between two-body and three-body interactions becomes significant; this explains the discrepancies between our two-body simulation results and experiments in this region. Apart from very small values of $\lambda\left(\mathrm{Au}_{1415} \mathrm{SC}_{2}\right.$, $\mathrm{Au}_{1415} \mathrm{SC}_{3}$, and $\mathrm{Au}_{561} \mathrm{SC}_{4}$ ), the OPM systematically overestimates the nearest-neighbor distance. The OPM also predicts a strong monotonic increase in the equilibrium distance with ligand length, which was only found to be correct for $3 \mathrm{D}$ structures $^{5}$ (for which the OPM was developed). However, the OPM provides no qualitative explanation for our numerical results on lower dimensional structures from Secs. III and IV.

We now consider the dependence of $\tau_{\text {eq }}$ on the capping density $\xi$. In our previous work, ${ }^{23}$ we have shown that when the capping layer is partially degraded due to ligand evaporation $(\xi=0.85)$, then the effective interaction becomes even more attractive and the equilibrium distance shifts very close to the fusion distance $\tau_{f}$. The latter is defined as the distance at which two NC cores touch each other; if the NC cores are not perfect spheres, $\tau_{f}$ is slightly larger than 1 . For icosahe- 
dral NCs, $\tau_{f} \approx 1.1$. We considered the systems $\mathrm{Au}_{147} \mathrm{SC}_{4}$ $(\lambda=0.56)$ and $\mathrm{Au}_{561} \mathrm{SC}_{4}(\lambda=0.37)$. The OCM yields for $\xi$ $=0.85$ the equilibrium distances in these systems $\tau_{\text {eq }}=1.15$ and $\tau_{\mathrm{eq}}=1.13$, respectively, both alarmingly close to $\tau_{f}$, in very good qualitative agreement with our simulation results. The estimates from the OPM (1.34 and 1.25), on the other hand, do not point to possible NC sintering.

In summary, the OCM quantitatively reproduces simulation and experimental data on monolayers in the region $0.35<\lambda<0.6$ and it explains why the equilibrium distance in a NC dimer is almost ligand length independent over a large range of $\lambda$. Moreover, the OCM explains the split between two-body simulation results and experiments for $\lambda>0.65$. Therefore, the OCM is the model of choice to describe low-dimensional NC superstructures, while for 3D superlattices the OPM can be applied.

\section{DISCUSSION}

We aim to combine the results of Secs. III-V with regard to ligand design for potential applications. Although the OCM is a purely geometric model, its results can be interpreted in terms of many-body interactions. We have seen in Sec. IV that the shift of the equilibrium distance in a $3-\mathrm{NC}$ system compared to a 2-NC case is correlated with the strength of the repulsive three-body interaction. Thus, we can consider the difference between the 2-NC and 3-NC or 4-NC OCM predictions as a qualitative measure for an energetic penalty associated with formation of $2 \mathrm{D}$ or $3 \mathrm{D}$ structures, respectively. This loss should of course be compared to the energetic gain due to a large number of contacts between NCs. Figure 12 shows that for long capping molecules the penalty may even overbalance the gain, so that self-assembly in 1D structures becomes energetically preferred over 2D close-packed ones, as found experimentally. ${ }^{3}$ In this section, we consider monodisperse NCs with $\xi=1$ (linear ligands, full capping) capped by a surfactant with scaled length $\lambda$. To give also a concrete example, we will write in brackets the corresponding range of alkylthiol ligands for a $\mathrm{NC}$ with $d_{c}$ $=5 \mathrm{~nm}$.

First, consider short ligands $\lambda \leq 0.39$ ( $\mathrm{SC}_{8}$ and shorter). The OCM predicts then the same equilibrium distance $\tau_{\text {eq }}$ [Eq. (18)] in 1D, 2D, and 3D assemblies, and thus no significant many-body effects are present. In this range, the NC superstructures with maximum coordination are very stable energetically, and the golden rule applies. Superlattices may readily precipitate from $\mathrm{NC}$ dispersion even without solvent evaporation. These superlattices are expected to form via the classical nucleation and growth mechanisms.

Next, consider ligands with intermediate length 0.39 $<\lambda \leq 0.65\left(\mathrm{SC}_{9}-\mathrm{SC}_{12}\right)$. In this regime, the OCM equilibrium distances between NCs in 1D and 2D aggregates are similar, while the spacing in $3 \mathrm{D} \mathrm{NC}$ aggregates is predicted to be larger and to increase systematically with ligand length. In particular, for $\lambda>0.54$ ( $\mathrm{SC}_{11}$ and longer), the entire space between $\mathrm{NC}$ cores is filled by ligand chains and NC-NC spacing $\tau_{\text {eq }}$ in 3D structures is predicted according to Eq. (19) with the suitable space filling factor $\eta$. The large difference between 2D and 3D allows us to estimate small to mod- erate three-NC interactions and large repulsive four-NC forces in this range of $\lambda$. Thus, the golden rule applies only to monolayers in this case, and they are energetically very stable. ${ }^{10}$ Due to the repulsive four-body interactions, the nucleation of 3D superlattices is expected to be hindered in one direction and their formation is predicted to occur via a layer-by-layer assembly. This may lead to a lattice distortion perpendicular to the substrate.

Finally, consider long ligands $0.65<\lambda<1.5$ $\left(\mathrm{SC}_{12}-\mathrm{SC}_{30}\right)$. In this case, the dependence of $\tau_{\text {eq }}$ on $\lambda$ in $1 \mathrm{D}$ and $2 \mathrm{D}$ structures is nonmonotonic. In $1 \mathrm{D}$ structures, the golden rule $\tau_{\mathrm{eq}} \approx 1.25$ is still obeyed. In $2 \mathrm{D}$ structures, on the other hand, the spacing between NCs is larger; in particular, the values of $\tau_{\text {eq }}$ remain in the interval $1.3 \leq \tau \leq 1.35$ over a relatively broad range of ligand lengths $0.8 \leq \lambda \leq 1.5$ $\left(\mathrm{SC}_{16}-\mathrm{SC}_{30}\right)$. This implies strong repulsive three-body interactions; as we have shown in Sec. IV, open 1D aggregates of NCs may even become energetically more favorable than $2 \mathrm{D}$ closed packed structures in this range of $\lambda$. This effect will inevitably introduce strain in NC superlattices, making the self-assembly into close-packed structures less favorable than more open and less ordered ones. ${ }^{3}$ Note that these long ligands form ordered bundles on the NC surface, especially at low temperatures. ${ }^{7,11,20}$ In our earlier work $^{23}$ we have shown that the equilibrium distance hardly changes upon transition through the melting temperature of these bundles. Therefore, the golden rule is still applicable.

We conclude that the capping molecules with $\lambda \leq 0.39$ are particularly suitable for formation of stable, robust $3 \mathrm{D}$ structures. Ligands with intermediate length $0.39<\lambda \leq 0.65$ are suited for creating stable close-packed monolayers. Long ligands can be used for spontaneous formation of open 1D structures such as chains, rings, or foams.

\section{CONCLUSIONS}

In summary, we computed and parameterized the PMF between alkylthiol capped gold NCs using atomistic simulations. The potential well depth is of the order of tens to hundreds $k_{B} T$. The minimum of the two-body PMF lies at $\approx 1.25 d_{c}$, suggesting a strong thermodynamic stability of $\mathrm{NC}$ structures with the ratio $\tau$ between the center-to-center distance and core diameter close to this value. We also investigated three-body effects on interactions between capped NCs in vacuum. If the capping layers of three NCs overlap pairwise, a repulsive three-body interaction is always present. Due to this interaction, the equilibrium distance in the two systems we studied shifted to $\approx 1.36 d_{c}$. The contribution of three-body effects to the total interaction energy is $20 \%$ for a short ligand and $40 \%$ for a long one. For long ligands, 1D (rings or chains) or fractal aggregates of capped NCs at the air-water interface are energetically more favorable than $2 \mathrm{D}$ ones (islands or close-packed monolayers). We introduced the OCM to explain our findings. This model considers the equilibrium distance between capped NCs as effective packing of flexible alkylthiol ligand tails. The OCM agrees well with our simulation results as well as with available experimental data on monolayers of capped NCs. We conclude that packing of ligands determines the spacing between NCs in 
aggregates, and interactions between capping layers play a crucial role in thermodynamic behavior and self-assembly of capped NC.

\section{ACKNOWLEDGMENTS}

T.J.H.V. acknowledges financial support from the Netherlands Organization for Scientific Research (NWO-CW) through a VIDI grant.

${ }^{1}$ S. Kinge, M. Crego-Calama, and D. N. Reinhoudt, ChemPhysChem 9, 20 (2008).

${ }^{2}$ R. P. Andres, J. D. Bielefeld, J. I. Henderson, D. B. Janes, V. R. Kolagunta, C. P. Kubiak, W. J. Mahoney, and R. G. Osifchin, Science 273 , 1690 (1996)

${ }^{3}$ J. R. Heath, C. M. Knobler, and D. V. Leff, J. Phys. Chem. B 101, 189 (1997).

${ }^{4}$ C. J. Kiely, J. Fink, M. Brust, D. Bethel, and D. J. Schiffrin, Nature (London) 396, 444 (1998).

${ }^{5}$ R. L. Whetten, M. N. Shafigullin, J. T. Khoury, T. G. Schaaff, I. Vezmar, M. M. Alvarez, and A. Wilkinson, Acc. Chem. Res. 32, 397 (1999).

${ }^{6}$ M. Brust, D. Bethell, D. J. Schiffrin, and R. Whyman, J. Chem. Soc., Chem. Commun. 1994, 801.

${ }^{7}$ U. Landman and W. D. Luedtke, Faraday Discuss. 125, 1 (2004).

${ }^{8}$ E. Shevchenko, D. Talapin, N. Kotov, S. O'Brien, and C. Murray, Nature (London) 439, 55 (2006).

${ }^{9}$ Z. Chen and S. O'Brien, ACS Nano 2, 1219 (2008).

${ }^{10}$ K. E. Mueggenburg, X.-M. Lin, R. H. Goldsmith, and H. M. Jaeger, Nature Mater. 6, 656 (2007).

${ }^{11}$ W. D. Luedtke and U. Landman, J. Phys. Chem. 100, 13323 (1996).

${ }^{12}$ K. Tay and F. Bresme, Mol. Simul. 31, 515 (2005).

${ }^{13}$ P. Atkins and J. de Paula, Physical Chemistry, 8th ed. (Oxford University Press, New York, 2006).

${ }^{14}$ D. Chandler, Introduction to Modern Statistical Mechanics, 1st ed. (Oxford University Press, New York, 1987).

${ }^{15}$ D. Frenkel and B. Smit, Understanding Molecular Simulations, 2nd ed. (Academic, San Diego, USA, 2002).

${ }^{16}$ P. Schapotschnikow, B. Hommersom, and T. J. H. Vlugt, J. Phys. Chem. C 113, 12690 (2009).

${ }^{17}$ J. Hautman and M. Klein, J. Chem. Phys. 91, 4994 (1989).

${ }^{18}$ P. Schapotschnikow, R. Pool, and T. J. H. Vlugt, Comput. Phys. Commun. 177, 154 (2007).

${ }^{19}$ R. Pool, P. Schapotschnikow, and T. J. H. Vlugt, J. Phys. Chem. C 111 10201 (2007)

${ }^{20}$ W. D. Luedtke and U. Landman, J. Phys. Chem. B 102, 6566 (1998).

${ }^{21}$ M. Lal, M. Plummer, N. J. Richmond, and W. Smith, J. Phys. Chem. B 108, 6052 (2004).

${ }^{22}$ N. Patel and S. A. Egorov, J. Chem. Phys. 126, 054706 (2007).

${ }^{23}$ P. Schapotschnikow, R. Pool, and T. J. H. Vlugt, Nano Lett. 8, 2930 (2008).

${ }^{24}$ T. J. H. Vlugt, R. Krishna, and B. Smit, J. Phys. Chem. B 103, 1102 (1999)

${ }^{25}$ D. Dubbeldam, S. Calero, T. J. H. Vlugt, R. Krishna, T. L. M. Maesen, and B. Smit, J. Phys. Chem. B 108, 12301 (2004).

${ }^{26}$ Y. Wang, S. Teitel, and C. Dellago, Chem. Phys. Lett. 394, 257 (2004).

${ }^{27}$ H. C. Hamaker, Physica 4, 1058 (1937).

${ }^{28}$ T. Ederth, Langmuir 17, 3329 (2001).

${ }^{29}$ H.-Y. Kim, J. O. Sofo, D. Velegol, M. W. Cole, and A. A. Lucas, Langmuir 23, 1735 (2007)
${ }^{30}$ G. Ciccotti, M. Ferrario, J. T. Hynes, and R. Kapral, Chem. Phys. 129, 241 (1989).

${ }^{31}$ E. Guàrdia, R. Rey, and J. A. Padró, Chem. Phys. 155, 187 (1991).

${ }^{32}$ M. G. Wolf, J. A. Jongejan, J. D. Laman, and S. W. de Leeuw, J. Am. Chem. Soc. 130, 15772 (2008)

${ }^{33}$ M. G. Wolf, J. A. Jongejan, J. D. Laman, and S. W. de Leeuw, J. Phys. Chem. B 112, 13493 (2008).

${ }^{34}$ J. Vreede, M. G. Wolf, S. W. de Leeuw, and P. G. Bolhuis, J. Phys. Chem. B 113, 6484 (2009).

${ }^{35}$ C. Jarzynski, Phys. Rev. Lett. 78, 2690 (1997).

${ }^{36}$ C. Jarzynski, Phys. Rev. E 56, 5018 (1997).

${ }^{37}$ S. Park, F. Khalili-Araghi, E. Tajkhorshid, and K. Schulten, J. Chem. Phys. 119, 3559 (2003).

${ }^{38}$ S. Park and K. Schulten, J. Chem. Phys. 120, 5946 (2004).

${ }^{39}$ N. Metropolis, A. W. Rosenbluth, M. N. Rosenbluth, A. H. Teller, and E. Teller, J. Chem. Phys. 21, 1087 (1953).

${ }^{40}$ J. Barker and R. Watts, Chem. Phys. Lett. 3, 144 (1969).

${ }^{41}$ J. I. Siepmann and D. Frenkel, Mol. Phys. 75, 59 (1992).

${ }^{42}$ D. Frenkel, G. C. A. M. Mooij, and B. Smit, J. Phys.: Condens. Matter 4, 3053 (1992).

${ }^{43}$ J. J. de Pablo, M. Laso, and U. W. Suter, J. Chem. Phys. 96, 6157 (1992).

${ }^{44}$ J. I. Siepmann, in Computer Simulation of Biomolecular Systems: Theoretical and Experimental Applications, edited by W. Van Gunsteren, P. Weiner, and A. Wilkinson (Escom, Leiden, 1993).

${ }^{45}$ W. C. Swope, H. C. Andersen, P. H. Berens, and K. R. Wilson, J. Chem. Phys. 76, 637 (1982).

${ }^{46}$ H. C. Andersen, J. Comput. Phys. 52, 24 (1983).

${ }^{47}$ J.-P. Ryckaert, G. Ciccotti, and H. J. C. Berendsen, J. Comput. Phys. 23, 327 (1977).

${ }^{48}$ T. Miller, M. Eleftheriou, P. Pattnaik, A. Ndrigano, D. Newns, and G. Martyna, J. Chem. Phys. 116, 8649 (2002).

${ }^{49}$ H. C. Andersen, J. Chem. Phys. 72, 2384 (1980).

${ }^{50}$ C. von Ferber, A. Jusufi, C. Likos, H. Löwen, and M. Watzlawek, Eur. Phys. J. E 2, 311 (2000)

${ }^{51}$ J. Dobnikar, M. Brunner, H. H. von Grünberg, and C. Bechinger, Phys. Rev. E 69, 031402 (2004).

${ }^{52}$ C. Russ, H. H. von Grünberg, M. Dijkstra, and R. van Roij, Phys. Rev. E 66, 011402 (2002)

${ }^{53}$ T. Terao, Mol. Phys. 104, 2507 (2006)

${ }^{54}$ D. Kofke and E. Glandt, Mol. Phys. 64, 1105 (1988).

${ }^{55}$ M. J. Hostetler, J. E. Wingate, C. Zhong, J. E. Harris, R. W. Vachet, M. R. Clark, J. D. Londono, S. J. Green, J. J. Stokes, G. D. Wignall, G. L. Glish, M. D. Porter, N. D. Evans, and R. W. Murray, Langmuir 14, 17 (1998).

${ }^{56}$ B. A. Korgel and D. J. Fitzmaurice, Phys. Rev. Lett. 80, 3531 (1998).

${ }^{57}$ B. A. Korgel, N. Zaccheroni, and D. Fitzmaurice, J. Am. Chem. Soc. 121, 3533 (1999).

${ }^{58}$ X. M. Lin, H. M. Jaeger, C. M. Sorensen, and K. J. Klabunde, J. Phys. Chem. B 105, 3353 (2001).

${ }^{59}$ M. B. Sigman, Jr., A. E. Saunders, and B. A. Korgel, Langmuir 20, 978 (2004).

${ }^{60}$ S. Huang, K. Minami, H. Sakaue, S. Shingubara, and T. Takahagi, Langmuir 20, 2274 (2004).

${ }^{61}$ A. V. Ellis, J. D'Arcy-Gall, K. Vijayamohanan, R. Goswami, P. G. Ganesan, C. Ryu, and G. Ramanath, Thermochim. Acta 426, 207 (2005).

${ }^{62}$ A.-I. Henry, A. Courty, M.-P. Pileni, P.-A. Albouy, and J. Israelachvili, Nano Lett. 8, 2000 (2008)

${ }^{63}$ J. E. Martin, J. P. Wilcoxon, J. Odinek, and P. Provencio, J. Phys. Chem. B 104, 9475 (2000).

${ }^{64}$ M. P. Pileni, J. Phys. Chem. B 105, 3358 (2001). 